PROCEEDINGS OF THE

AMERICAN MATHEMATICAL SOCIETY

Volume 133, Number 9, Pages 2489-2490

S 0002-9939(05)08138-4

Article electronically published on April 8, 2005

\title{
HYPERBOLIC GROUPS HAVE FINITE ASYMPTOTIC DIMENSION
}

\author{
JOHN ROE
}

(Communicated by Mohan Ramachandran)

\begin{abstract}
We detail a proof of a result of Gromov, that hyperbolic groups (and metric spaces) have finite asymptotic dimension. This fact has become important in recent work on the Novikov conjecture.
\end{abstract}

\section{INTRODUCTION}

Let $X$ be a metric space, with basepoint $x_{0}$. We use the notation $|x|$ to denote $d\left(x, x_{0}\right)$. If $x, y \in X$, then the Gromov product $(x \mid y)$ is the positive real number $\frac{1}{2}(|x|+|y|-d(x, y))$. By definition [3], $X$ is hyperbolic if there is $\delta>0$ such that

$$
(x \mid z) \geq \min \{(x \mid y),(y \mid z)\}-\delta
$$

for all $x, y, z \in X$.

Let $\mathcal{U}$ be a family of subsets of $X$. We say that $\mathcal{U}$ is $d$-disconnected if the minimum distance between any two distinct sets of the family $\mathcal{U}$ is at least $d$. We say that $\mathcal{U}$ is $r$-bounded if each set in the family has diameter $\leq r$. One says that $X$ has finite asymptotic dimension if there is a number $N$ such that for each $d>0$ there is an $r>0$ such that $X$ can be covered by at most $N+1 d$-disconnected, $r$-bounded families. The least such $N$ is the asymptotic dimension of $X$.

This definition is due to Gromov [4, page 29] and was crucial to the work of $\mathrm{Yu}$ [5] on the Novikov conjecture. On page 31 of [4], Gromov remarks that word hyperbolic groups have finite asymptotic dimension. Below we present a short proof of (a slight generalization of) this result. Our proof is related to those of the finite-dimensionality of the Gromov boundary of a hyperbolic group given in [2] and [1].

\section{THE PROOF}

Let $X$ be a geodesic metric space. Say that $X$ has bounded growth if for each $s>0$ there is a number $N_{s}$ such that each ball of radius $S+s$ in $X$ can be covered by at most $N_{s}$ balls of radius $S$.

Since $X$ is geodesic, one may take for $N_{s}$ the supremum (if finite) of the cardinalities of $s$-separated subsets in balls of radius $2 s$. This observation shows that a

Received by the editors May 1, 2002.

2000 Mathematics Subject Classification. Primary 20F67; Secondary 55M10.

Key words and phrases. Gromov hyperbolicity, coarse geometry, asymptotic dimension.

The author was supported in part by NSF Grant \#0100464.

(C)2005 American Mathematical Society Reverts to public domain 28 years from publication 
space of bounded geometry has bounded growth. In particular, the Cayley graph of a finitely generated group has bounded growth.

Theorem 2.1. Let $X$ be a hyperbolic geodesic metric space with bounded growth. Then $X$ has finite asymptotic dimension.

Proof. Fix a basepoint $x_{0} \in X$, and let $d>0$ be given. Suppose that $X$ is $\delta$ hyperbolic. Let $A_{k}$ denote the annulus $\{x \in X: k d \leq|x| \leq(k+1) d\}$ in $X$. It will suffice to show that there is a number $N$, independent of $d$, such that each annulus $A_{k}$ can be covered by a family of sets $\left\{U_{i}\right\}$, each having diameter no more than $4 d+4 \delta$, and such that no more than $N$ of the sets $U_{i}$ have nonempty intersection, in $A_{k}$, with any set of diameter $d$.

Let $\left\{x_{i}\right\}$ be a maximal $d$-separated subset of the sphere $\{x:|x|=k d\}$ of radius $k d$ and define $U_{i}=\left\{x \in A_{k}:\left(x \mid x_{i}\right) \geq\left(k-\frac{1}{2}\right) d-\delta\right\}$. If $x \in A_{k}$ let $x^{\prime}$ denote the point where a geodesic from $x_{0}$ to $x$ intersects the sphere of radius $k d$. Then $\left|x^{\prime}\right|=$ $\left(x \mid x^{\prime}\right)=k d$. By maximality there is some $i$ for which $d\left(x^{\prime}, x_{i}\right) \leq d$ and therefore $\left(x^{\prime} \mid x_{i}\right) \geq\left(k-\frac{1}{2}\right) d$. By hyperbolicity $\left(x \mid x_{i}\right) \geq \min \left\{\left(x \mid x^{\prime}\right),\left(x^{\prime} \mid x_{i}\right)\right\}-\delta \geq\left(k-\frac{1}{2}\right) d-\delta$ and so $x \in U_{i}$. Thus the $U_{i}$ cover $A_{k}$ as asserted.

Suppose $x \in U_{i}$. Then $d\left(x, x_{i}\right)=|x|+\left|x_{i}\right|-2\left(x \mid x_{i}\right) \leq 2 d+2 \delta$. Thus the $U_{i}$ have uniformly bounded diameter.

Suppose that $U_{i}$ meets the ball of radius $d$ around some $x \in A_{k}$; let $y$ be a point in the intersection. Let $x^{\prime \prime}$ be the point where a geodesic ray from $x_{0}$ to $x$ intersects the sphere of radius $\left(k-\frac{1}{2}\right) d$, so that $\left|x^{\prime \prime}\right|=\left(x \mid x^{\prime \prime}\right)=\left(k-\frac{1}{2}\right) d$. We also have $(x \mid y) \geq\left(k-\frac{1}{2}\right) d$, and $\left(x_{i} \mid y\right) \geq\left(k-\frac{1}{2}\right) d-\delta$, so $\left(x_{i} \mid x^{\prime \prime}\right) \geq\left(k-\frac{1}{2}\right) d-3 \delta$. It follows that $d\left(x_{i}, x^{\prime \prime}\right)=\left|x_{i}\right|+\left|x^{\prime \prime}\right|-2\left(x_{i} \mid x^{\prime \prime}\right) \leq \frac{1}{2} d+6 \delta$. The maximum number of $U_{i}$ that meet the ball of radius $d$ around $x$ is therefore bounded by the maximum cardinality of a $d$-separated subset in a ball of radius $\frac{1}{2} d+6 \delta$. But this cardinality is bounded by the number $N_{6 \delta}$ arising from the definition of bounded growth. The proof is complete.

\section{REFERENCES}

1. M. Bonk and O. Schramm, Embeddings of Gromov hyperbolic spaces, Geometric and Functional Analysis 10 (2000), 266-306. MR1771428 (2001g:53077)

2. E. Ghys and P. de la Harpe, Sur les groupes hyperboliques d'après Mikhael Gromov, Progress in Mathematics, vol. 83, Birkhäuser, Boston, 1990. MR,1086648 (92f:53050)

3. M. Gromov, Hyperbolic groups, Essays in Group Theory (S.M. Gersten, ed.), Springer-Verlag, New York-Heidelberg-Berlin, 1987, Mathematical Sciences Research Institute Publications 8, pp. 75-263. MR0919829 (89e:20070)

4. _ Asymptotic invariants for infinite groups, Geometric Group Theory (G.A. Niblo and M.A. Roller, eds.), LMS Lecture Notes, vol. 182, Cambridge University Press, Cambridge, 1993, pp. 1-295. MF 1253544 (95m:20041)

5. G. Yu, The Novikov conjecture for groups with finite asymptotic dimension, Annals of Mathematics 147 (1998), 325-355. MR 1626745|(99k:57072)

Department of Mathematics, Penn State University, University Park, Pennsylvania 16802

E-mail address: roe@math.psu.edu 